\title{
Survey on Project Management System using Event based Scheduler and Ant Colony Optimization
}

\author{
R. S. Vairagade \\ Savitribai Phule Pune \\ University \\ Sits \\ Narhe,Pune-411041
}

\author{
Rohan Arora \\ Savitribai Phule Pune \\ University \\ Sits \\ Narhe,Pune-411041
}

\author{
Vinita Gaikwad \\ Savitribai Phule Pun \\ University \\ Sits Narhe, \\ Pune-411041
}

\author{
Divyansh Singh \\ Savitribai Phule Pune \\ University \\ Sits \\ Narhe,Pune-411041
}

\author{
Prachi Jadhav \\ Savitribai Phule Pune \\ University \\ Sits \\ Narhe,Pune-411041
}

\begin{abstract}
Resource allocation and tasks assignment to software development teams are very crucial and arduous activities that can affect a project's cost and completion time. Solution for such problem is NP-Hard and requires software managers to be supported with efficient tools that can perform such allocation and can resolve the software development project scheduling problem (SDPSP) more efficiently. Ant colony optimization (ACO) is a rapidly evolving meta-heuristic technique based on the real life behavior of ants and can be used to solve NP-Hard (SDPSP) problem.

Different versions of ACO meta-heuristic have already been applied to the software project scheduling problem in the past that took various resources into account. We have applied elitist strategy of ACO (elitist ant system) for solving SDPSP in a parameter-constrained environment taking project's cost and duration into consideration. The objective of the ACOSDPSP methodology allows software project managers and schedulers to assign most effective set of employees that can contribute in minimizing cost and duration of the software project. Experimental results show that the proposed ACOSDPSP methodology is promising in achieving the desired results.
\end{abstract}

\section{General Terms}

Simulation,Data dictionary/directory ,Data warehouse and repository,Logging recovery,Security, integrity and protection

\section{Keywords}

PERT: Program Evaluation and Review Technique

CPM :Critical Path Method

RCPSP: Resource Constrained Project Scheduling Problem

\section{EBS: Event Based Scheduler}

ACO:Ant Colony Optimization

\section{INTRODUCTION}

Software projects are people-intensive activities and their related resources are mainly human resources. Thus, an adequate model for software project planning has to deal with not only the problem of project task scheduling but also the problem of human resource allocation. But as both of these two problems are difficult, existing models either suffer from a very large search space or have to restrict the flexibility of human resource allocation to simplify the model. Different software project tasks require employees with different skills, and skill proficiency of employees significantly influences the efficiency of project execution. As such, assigning employees to the best-fitted tasks is challenging for software project managers, and human resource allocation has become a crucial part in software project planning. Techniques like PERT and CPM lack the consideration of resource allocation and scheduling models like the RCPSP do not consider the allocation of employees with various skills. Therefore, this technique usually regard task scheduling and human resource allocation as two separated activities and leave the job of human resource allocation to be done by project managers manually so resulting is inefficient resource allocation and poor management performance

\section{RELATED WORK}

\subsection{Use of genetic algorithms}

C. K. Chang et al. [3] proposed Genetic Algorithms (GAs) for project scheduling, in which weights are assigned to edges of Task Precedence Graph. Then it selects two parent solutions and swaps over feature from each to solve task scheduling problem in software project management. It calculates the complexity of each solution and selects the weighted objective function to get single solution. This helps the engineer to 
work with bug free and reliably. But continuity of allocation is not supported. Carl K. Chang et al. [4], proposed a Software Project Management Net (SPMNet) to model software development projects. It helps to allocate the resources and can do project scheduling automatically based on the skills and experience. It creates plan to develop and estimates the schedules and budgets.

\subsection{Optimized Based Approach}

Ahilton Barreto et al. [6], proposed an optimized based approach to support resource allocation of a software project which includes project activities, human resources available. In this approach, some of the professionals are taken into consideration with their available schedules, Maximum cost and Minimum cost for their working hours. The goal is to allocate the cheapest team for the project. Each team is assigned for same project to find the efficient and cheap team. But in this approach, the study states that there is no difference between the time to perform the activity and to choose the team. An effective and efficient automated acyclic Task Precedence Graph is required for identifying Task priority and allocation of suitable employees to tasks based on their skills and experience with least cost. In the proposed technique Event Based Scheduling and Ant Colony

Optimization techniques are used to provide software project Scheduling and human resource allocation.

\subsection{Max-Min Ant System}

Thomas Stutzle et al. [5], proposed Max-Min Ant System (MMAS) for travelling salesperson problem. To exploit the best solutions during the search and direct the ants to high quality solutions. In this, the Max-Min ant system will limit the pheromone trail to prevent premature convergence. The MMAS failed to manage the best managing solutions of pheromone trails. This technique is included in the proposed approach by rectifying the previous problem to manage the best managing solutions.

\section{MODEL DESCRIPTION}

In existing project management (PM) techniques, commercial PM tools, and research prototypes in not efficient in computational capabilities and only provide passive project tracking and reporting aids. Project managers must make all major decisions based on their individual insights and experience and must build the project database to record such decisions and produce reports in various formats such as Gantt or Pert charts. Marco Dorigo proposed a new optimization technique called Ant Colony Optimization (ACO). Main characteristics of this model are positive feedback, distributed computation, and the use of a constructive greedy heuristic. Positive feedback accounts for rapid discovery of good solutions, distributed computation avoids premature convergence, and the greedy heuristic helps find acceptable solutions in the early stages of the search process. The algorithms they developed are models derived from the study of real ant colonies and are called Ant algorithms. Liu and Wang [1] developed a flexible model for handling the optimization of scheduling problems in linear construction projects involving different objectives and resource argument tasks. The model is suggested for the scheduling of construction activities of high-rise building or bridge projects. Jianxing and Cang [2] demonstrated the use of ant colony optimization algorithm to solve the dynamic problem of resource scheduling in group project management.

\section{Ant Colony Optimization}

The suggested strategy is defined by two most important features. First, a description scheme made from activity list along with a planned employee allotment matrix together with a novel event based scheduler takes shape. It allows the modeling of resource conflict and activity preemption. Second, an ACO strategy is suggested since it shows successful application to varied combinatorial maximization issues. ACO builds solutions inside a step-by-step manner that will actually make $d$ ants to plan the critical tasks far back as possible and then to assign the construction project tasks to appropriate employees with required skills. The suggested method efficiently manages employees using an employee database and it also identifies tasks utilizing an Activity Precedence Graph which defines than a activity are only able to start when all of that direct predecessor tasks have finished. Hence the planning objective among the suggested strategy is promising. The suggested system will reduce overall project cost, resources are resourcefully employed in this task as well as a new technique for resolving the software program project planning problem It certainly will decrease the two basic issues in software project management that might be activity scheduling and employee allotment. They provide the clear idea for time scheduling and resource allotment and is going to lessen the manual effort. The suggested system utilize the resources efficiently and allowing the employers to finalize anyone activity among the given time. It furnishes one of the best way to solve this activity scheduling and employee allotment issues in software project management process. Reckoning on the worker expertise, allocating employee to certain activity resource permit the construction project to become completed promptly. By evaluating employees work time that is undoubtedly per hour salary for normal effort and overtime the expense of this project can possibly be minimized. The suggested system helps Project manager in allocating projects to Team leaders and in its place for Team leaders for allotment of activity to team members. It aids allocate employee to perform in overtimes to deal with their tasks as well as having the suggested algorithm is able to yield better plans with lower costs, stronger workload assignments decreases the dimensions of the request space in contrast to other existent approaches.

\section{ACO ALGORITHM}

1. Initialize all parameters i.e. Q0, Ngen, Nant which are used in ACO. These parameters are used to evaluate the importance of Heuristic information and history, which also adjust the pheromone updating, balance the behavior of ants. Nant is number of ants and Ngen is number of generations.

2. Initialize all pheromone value as 0 .

3. Each ant selects her own path for finding solution. Each ant select next node as per selection scheme and fill the matrix. When travelling of ants is completed Solution matrix is constructed.

4. By using fitness function evaluates the quality of solution, also calculate the cost, duration of project and overwork for that project.

5. Compare the solutions and select the best one, update the pheromone value.

6. Repeat the procedure till condition is satisfied. Generally termination condition is determined by fix number of generation.

7. Select and display the best solution whose cost and duration less. 


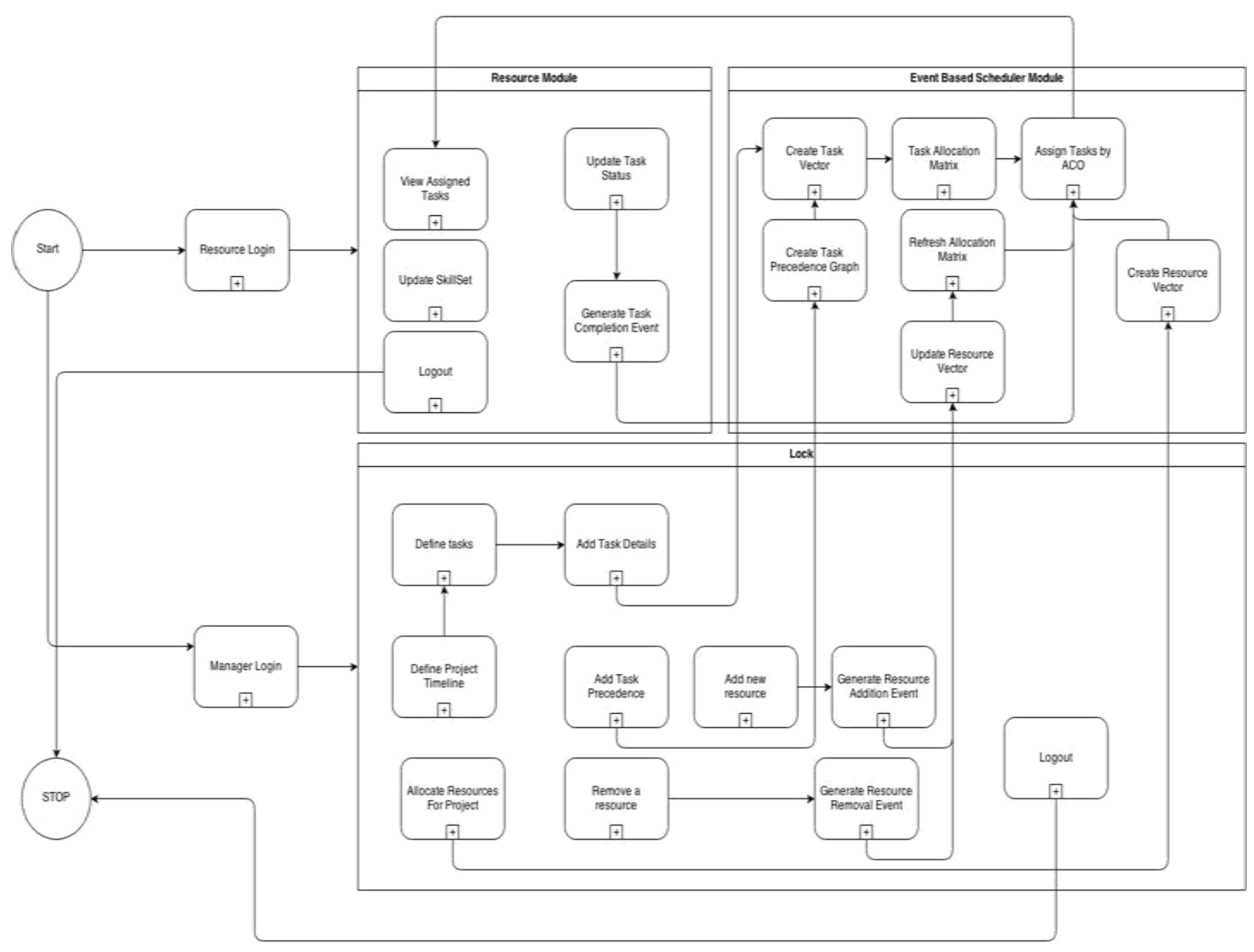

Fig 1: Detailed architectural diagram

\section{LITERATURE SURVEY \\ 4.1 The Resource Constrained Project Scheduling Problem Model}

The RCPSP is a classic model in project management which is NP-hard [10], [15]. It involves scheduling the tasks of a project subject to precedence and resource constraints. To build a schedule, one needs to determine an order of tasks which forms a task list. The task list defines the tasks' priorities to consume resources when resource conflict occurs [10].Although the RCPSP is important and widely used, it only focuses on the issue of project scheduling and does not take the issue of employee allocation into account. Therefore, the RCPSP is still inadequate for modeling the software project planning problem.

\subsection{Employee Allocation Models}

Employee allocation problems have attracted a considerable amount of research effort in recent years and various models like nurse rostering [16] and personnel planning[17], [18] have been proposed. There are also some studies that focus on the employee allocation problem in software projects [19], [20], [21], [22], [23]. In these models, the problem of how to assign employees to different workstations (or tasks/time periods) is addressed. The optimization objective is to minimize the number of constraint violations [10], [11], [12] or to minimize project duration and cost [19], [20], [21], [22], [23].However, they lack the consideration of task scheduling. To plan a software project, the project manager has to decide not only the allocation of human resources but also the start and finish time of each task. As the working duration of a task is dependent on the employees assigned to the task, separating task scheduling and employee allocation as two independent events is unsuitable.

\subsection{Multiskill Scheduling Model}

Considering the fact that software development involves a group of employees with different skills, Bellenguez and Ne'ron [19], [20] extended the traditional RCPSP model to propose a multi skill project scheduling model for software project planning. The model follows the framework of the RCPSP for task scheduling and regards different combinations of employees as different alternative modes for the implementation of a task. RCPSP and couples the modeling of employees with various skills, it provides a more practical way for software project planning as it can deal with both the problems of task scheduling and employee allocation. But, since the model assumes that an employee can only be assigned to a task at one time and no task preemption is allowed, the flexibility of human resource allocation is reduced. Under such restriction, if any one of the employees assigned to a task is busy with other activities, the whole team has to wait until that employee is released. This situation may reduce the resource efficiency of the project.

\subsection{Time-Line-Based Model}

To model the task scheduling and human resource allocation problem in a more flexible way, Chang et al. [11] considered task scheduling and employee allocation together and developed a time-line-based model. The $3 \mathrm{D}$ representation is able to overcome the disadvantages of the RCPSP and the employee allocation models. But it also brings in two newproblems. First, as workloads are assigned period-by- 
period instead of task-by-task, the plans produced by this model may assign two completely different groups of employees to the same task in different periods. As a result, the task may be implemented in adesultory manner, which is inefficient. Second, as the representation size of a schedule is enlarged from a 2Dmatrix to a 3D one, the search space of the problem significantly increases.

\section{CONCLUSION}

The main objective of this software is first, the method takes advantage of ACO to solve the complicated planning problem, the second one method introduces an event-based scheduler. Both methods have limitation during the project planning and allocation. Experimental results show that the representation scheme with the EBS is effective in small target tasks, and the ACO algorithm manages to yield better plans with high statisticts and mean access time and more stable workload assignments compared with other existing approaches. A new method for solving the software project planning problem has been proposed in future work. The problem of task preemption exists in the previous models. The existing system also suffers from the problem of allocating the same task for different group of employees in different periods. ACO solve the problem of project scheduling but it does not consider the employee allocation matrix. The ACO is not a satisfactory model to solve the problem of project scheduling.

\section{REFERENCES}

[1] Vahid Khodakarami, Norman Fenton, Martin Neil, "Project Scheduling:Imposed Approach to Incorporate Uncertainty using Bayesian Networks"(2007)".

[2] Carl K.Chang,Hsin-Yi Jiang,Yu Di,Dan Zhu,Yujia Ge,"Time-line based model for software project scheduling with genetic lgorithms"(2008)

[3] C.K. Chang, H. Jiang, D. Zhu, Y. Di, and Y. Ge, "Timeline Based Model for Software Project Scheduling with Genetic Algorithms", Information and Software Technology, vol. 50, pp. 1142-1154, 2008.

[4] C.K. Chang, C. Chao, M.J. Christensen, and T.T. Nguyen, "Software Project Management Net: A New Methodology on Software Management", Proc. 22nd Ann. Int ${ }^{\mathrm{e}} 1$ Computer Software and Applications Conf., 1998.

[5] T. Stutzle and H. Hoos, "Max-Min Ant System", Future Generation Computer Systems, vol. 16, no. 8, pp. 889$914,2000$.

[6] A. Barreto, C.M.L. Werner, "Staffing a Software Project: A Constraint Satisfation and Optimization-Based approach", Computers and Operations Research, vol. 25, pp. 3073-3089, 2008.

[7] B. Boehm, Software Engineering Economics. PrenticeHall, 1981.

[8] B. Boehm et al., Software Cost Estimation with COCOMO II. Prentice-Hall, 2000.

[9] A. Shtub, J.F. Bard, and S. Globerson, Project Management: Processes, Methodologies, and Economics, second ed. Prentice Hall, 2005.

[10] P. Brucker, A. Drexl, R. Mohring, K. Neumann, E.
Pesch, "Resource-Constrained Project Scheduling: Notation, Classification, Models and Methods," European J. Operational Research, vol. 112, pp. 3-41, 1999.

[11] C.K. Chang and M. Christensen, "A Net Practice for Software Project Management," IEEE Software, vol. 16, no. 6, pp. 80-88, Nov./Dec. 1999.

[12] C.K. Chang, M.J. Christensen, C. Chao, and T.T. Nguyen, "Software Project Management Net: A New Methodology on Software Management," Proc. 22nd Ann. Int'l Computer Software and Applications Conf., 1998.

[13] A. Kumar V.K. and L.S. Ganesh, "Use of Petri Nets for Resource Allocation in Projects," IEEE Trans. Eng. Management, vol. 45, no. 1, pp. 49-56, Feb. 1998.

[14] L.C. Liu and E. horowitz, "A Formal Model for Software Project Management," IEEE Trans. Software Eng., vol. 15, no. 10, pp. 1280-1293, Oct. 1989.

[15]L. Ozdamar, "A Genetic Algorithm Approach to a General Category Project Scheduling Problem," IEEE Trans. Systems, Man, and Cybernetics-Part C: Applications and Rev., vol. 29, no. 1, pp. 44-59, Feb. 1999.

[16] R. Bai, E.K. Burke, G. Kendall, J. Li, and B. McCollum, "A Hybrid Evolutionary Approach to the Nurse Rostering Problem," IEEE Trans. Evolutionary Computation, vol. 14, no. 4, pp. 580-590, Aug. 2010.

[17] V. Nissen and M. Gu" nther, "Automatic Generation of Optimised Working Time Models in Personnel Planning," Proc. Seventh Int'l Conf. Swarm Intelligence, pp. 384-391, 2010.

[18] M. Gu" nther and V. Nissen, "Particle Swarm Optimization and an Agent-Based Algorithm for a Problem of Staffing Scheduling," Proc. Int'l Conf. Applications of Evolutionary Computation, pp. 451-461, 2010.

[19] C.K. Chang, M.J. Christensen, and T. Zhang, "Genetic Algorithms for Project Management," Annals of Software Eng., vol. 11, pp. 107-139, 2001.

[20]G. Antoniol, M. Di Penta, and M. Harman, "Search-Based Techniques Applied to Optimization of Project Planning for a Massive Maintenance Project," Proc. 21st IEEE Int'1 Conf. Software Maintenance, 2005.

[21] G. Antoniol, M. Di Penta, and M. Harman, "SearchBased Techniques for Optimizing Software Project Resource Allocation," Genetic and Evolutionary Computation, vol. 3103, pp. 1425-1436, 2004.

[22] Y. Ge, "Software Project Rescheduling with Genetic Algorithms," Proc. Int'l Conf. Artificial Intelligence and Computational Intelligence, 2009.

[23] M. Dorigo, V. Maniezzo, and A. Colorni, “Ant System: Optimization by a Colony of Cooperating Agents," IEEE Trans. Systems Man, and Cybernetics-Part B: Cybernetics, vol. 26, no. 1, pp. 29-41, Feb. 1996 